\title{
Dynamic earth pressures against a retaining wall caused by Rayleigh waves
}

\author{
C. Navarro and A. Samartin
}

An approximate procedure for studying harmonic soil-structure interaction problems is presented. The presence of Rayleigh waves is considered and the resulting governing equations of the dynamic soil-structure system are solved in the time domain. With this method the transient and steady states of a vibratory motion and also the nonlinear behaviour of the soil can be studied.

As an example, the dynamic earth pressure against a rigid retaining wall is investigated. The loads are assumed to be harmonic Rayleigh waves with both static and dynamic surface surcharges. The dependence of the results on the excitation frequency is shown.

Keywords: soil dynamics, soil-structure interaction, dynamic earth pressures, retaining walls

Among the different problems of dynamic soil-structure interaction, those related to harmonic excitation are very important. Examples of this type of problem are vibrations induced by rotating machinery, soil-structure systems with a predominant mode of vibration.

Finite element methods of analysing soil-structure interaction problems can be divided according to the way they formulate the governing equations of motion, namely time-domain analysis and frequency-domain analysis. It is also possible to use both types of analyses simultaneously, i.e. a hybrid or mixed method. An excellent review of dynamic soil-structure interaction using the finite element method has been reported by Bayo and Wilson ${ }^{1}$.

The choice of the method to be applied to a particular problem depends on the type of expected response. So, in frequency-domain analysis, only structurally linear problems in a stationary regime can be studied. In time-domain analysis, apart from these problems, geometric and material nonlinear cases in a transient regime can also be solved. However, a time-domain analysis involves much greater computational effort than a frequency-domain analysis and is therefore a more expensive solution. This last aspect is becoming less important with current trends in the computer industry and the advances in numerical analysis techniques.

As an alternative to modelling very large soil volumes, special boundaries such as non-reflecting or absorbing types, are used in the finite element analysis of dynamic soil-structure interaction problems. This avoids unrealistic wave reflections against the artificial boundaries introduced in the mathematical model and, thus, the results are not distorted. In the frequency domain a complete formulation of this type of boundary was presented by Waas ${ }^{2}$. However, such a boundary does not exist for time-domain analyses.

A brief review of this situation is given by Navarro and Samartin ${ }^{3}$ in which a time-domain boundary, derived from the Waas boundary, is applied to the solution of harmonic, soil-structure interaction problems. Analyses have also been made accounting for the nonlinear behaviour of the soil mass below the structure, by using the above-mentioned viscoelastic transmitting boundary (Navarro and Samartin ${ }^{4}$ ). These last analyses show the need for solving soil-structure interaction problems in the time domain. Another important question in the modelling of soil-structure interaction problems is how to introduce the dynamic excitation. There are two main possibilities. The first is to impose timedependent displacements at a given set of nodes of the finite element mesh (kinematic boundary conditions). This methodology is used in the analysis of some soil-structure interaction problems, when shear and compressional waves are propagating vertically and a clear soil-hardrock interface can be defined. In this case, a control motion, obtained by a deconvolution process from a given 
surface motion, is imposed at nodes of the interface. All incident energy generated into the model is reflected in the soil-rock contact beside the finite element mesh. This approach becomes unrealistic when interfaces are not clearly defined, i.e. when a deep soil deposit is involved in the analysis (no horizontal interface), or Rayleigh waves, propagating through horizontally undefined soil layers, are considered (no vertical interface). As an alternative to the first methodology, a set of forces prescribed at selected nodes of the model edges (inertial boundary conditions), with appropriate transmitting or absorbing boundaries, can be imposed. The system of introduced forces has to produce free-field displacements. This last procedure will be described in a later section.

Both approaches are equivalent for free-field conditions, i.e. without structures. However, the presence of structures will produce waves that will progagate through the modelled soil and reflect against the boundaries with prescribed displacements. These reflections can be avoided by using non-reflecting boundaries with prescribed forces.

\section{Non-reflecting boundary in the time domain}

Different approaches are used to solve dynamic problems involving infinite domains. However, those related to the use of transmitting and absorbing boundaries offer the possibility of reducing the finite element mesh size, with economical and computational savings. The use of alternative techniques, such as the boundary element method, can also be attractive.

The problem of using non-reflecting conditions is satisfactorily solved by $\mathrm{Waas}^{2}$ for frequency-domain analysis. This boundary condition is frequency dependent and has been used in some computer codes, such as FLUSH (Lysmer et $a l^{5}$.) and CREAM (Gómez-Massó6), for soil-structure interaction analysis in the frequency domain.

For time-domain analysis, satisfactory non-reflecting boundaries, similar to the consistent transmitting boundary developed by $\mathrm{Waas}^{2}$ for frequency-domain analysis, have not yet been obtained. However, important progress in this direction has been reported by Zienkiewicz et al ${ }^{7}$. and $\operatorname{Shen}^{8}$, who have presented a method based on the transmitting boundary developed by Smith ${ }^{9}$. A comparison of the different behaviour of transmitting boundaries in time domain was offered by Wolf ${ }^{10}$.

The non-reflecting boundary for time-domain soilstructure interaction problems used in this paper is obtained, from the consistent transmitting boundary developed by Waas ${ }^{2}$, in the following way: let $K(\omega)$ be the consistent stiffness matrix at frequency $\omega$ which represents the 'exact' relationship between forces $P \mathrm{e}^{\mathrm{i} \omega t}$ and displacements $D \mathrm{e}^{\mathrm{i} \omega t}$ at all degrees of freedom existing along the edge where consistent transmitting boundary is acting. The impedance matrix $K(\omega)$ can be found by considering the propagation of plane horizontal waves in a stationary regime through a horizontally layered soil. Details of the computations are described by Waas ${ }^{2}$ and Tassoulas and Kausel ${ }^{11}$.

For the time domain analysis, a similar boundary condition can be considered as follows ${ }^{3}$ :

$$
P(t)=K D(t)+C \dot{D}(t)
$$

where

$$
\begin{aligned}
& K=\operatorname{Re}\{K(\omega)\} \\
& C=\frac{1}{\omega} \operatorname{Im}\{K(\omega)\}
\end{aligned}
$$

and $P(t), D(t)$ and $\dot{D}(t)$ are the force, displacement and velocity vectors at all degrees of freedom existing along the boundary.

This last boundary condition simulates the exact dynamic effects of the semi-infinite viscoelastic horizontally layered soil system beyond the modelled region when a harmonic wave of frequency $\omega$ is acting on it. Thus this transmitting boundary can in principle be placed very near the structure and offers, in relation to the Smith boundary ${ }^{9}$ and other techniques ${ }^{7,8}$ based on it, the possibility of greatly reducing the mesh size and the computational time for harmonic problems because only a slight increment of the bandwidth of the structural matrices is necessary. Additionally, the proposed boundary condition allows an easier treatment of Rayleigh wave input, as will be observed later. However, two important aspects must be pointed out. The first refers to multi-frequency excitation, i.e. seismic excitation. In this case, a careful choice of the transmitting boundary frequency must be made, as is shown by Navarro and Samartin ${ }^{14}$ and Alheid et al. ${ }^{12}$, but it seems reasonable to obtain the transmitting boundary for a frequency equal to the highest significant frequency of the involved excitation ${ }^{4}$. The second aspect refers to the behaviour of the time-domain consistent transmitting boundary in the transient phase of the vibratory motion. In fact, the impedance matrix $K(\omega)$ was obtained by Waas ${ }^{2}$ in the steady state of the process, supposing that only planar waves were acting on the transmitting boundary. To study the approximation of the boundary when a transitory regime and non-planar waves are acting, the example shown in Figure la is studied. This case corresponds to the vertical forced vibration of a rigid strip founded on a soil with one layer whose depth $H$ is equal to the width strip $2 B$.

The constants of the viscoelastic soil are: $G=18000 \mathrm{t} /$ $\mathrm{m}^{2}$ (shear modulus), $\beta=300 \mathrm{~m} / \mathrm{s}$ (shear wave velocity), $v=0.4$ (Poisson ratio) and $\delta=5 \%$ (damping ratio). Two finite element meshes are used (Figure 2) and they correspond to the situations shown in Figures $l b$ and $l c$, respectively. The finite elements used in the analysis are isoparametric rectangles with four nodes. The total number of elements is 17 in the first model and 387 in the second. In both cases the symmetry characteristics of the problem have been taken into account. In the first
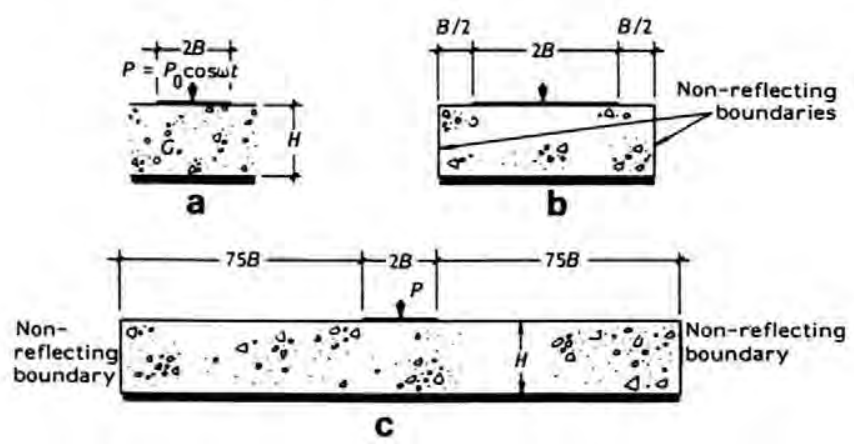

Figure 1 Non-reflecting boundary situation 


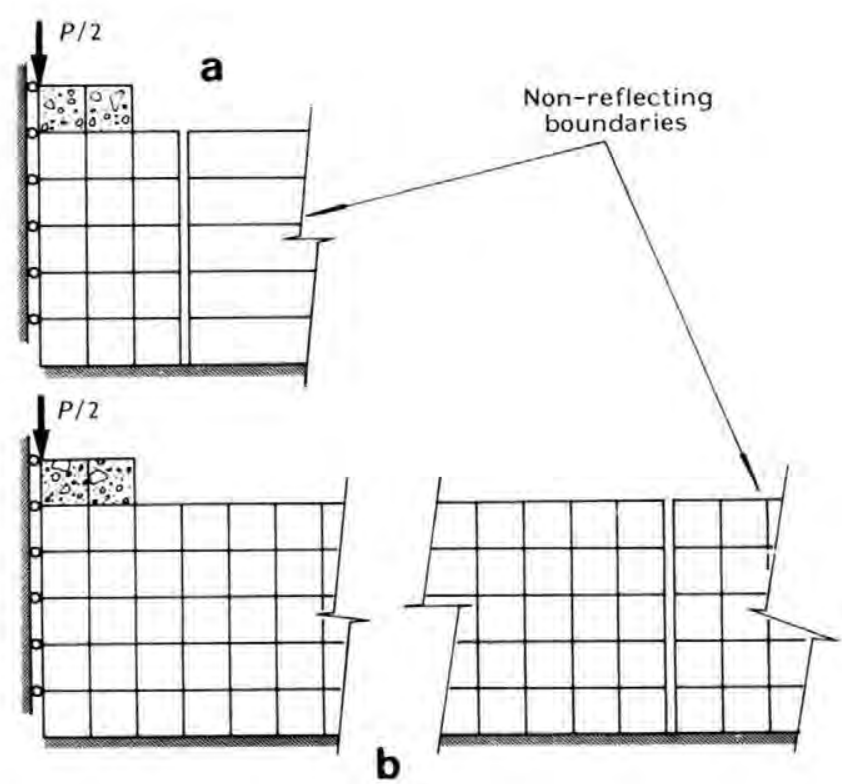

Figure 2 Finite element models

model the aforementioned boundary element is used. With the second model, the boundary is placed sufficiently removed from the foundation to avoid the reflecting wave phenomena already mentioned during the time analysis.

The time domain analysis is made by using a time step $\Delta t=T / 32$, where $T$ is the period of the excitation force. A wide range of input frequencies, defined by the dimensionless frequency $a_{0}$, is studied. The following notation is used

$$
a_{0}=\omega B / \beta
$$

where $\omega=$ is the frequency of the input force, $B$ is the half-width of the strip and $\beta$ is the propagation velocity of the shear waves.

For this problem, it has been shown elsewhere ${ }^{3}$ that when the dimensionless frequency $a_{0}$ is greater than two, the transient response, considered as the vertical displacement of the rigid strip, can reach values significantly larger than those observed in the stationary phase of the process. To test the behaviour of the consistent transmitting boundary in time domain, three values $(2.0,2.25$ and 2.50$)$ for the parameter $a_{0}$ are considered.

In Figure 3 the response time-history of the normalized vertical displacement of the foundation is given for each value of $a_{0}$ according to the model used. The similarity

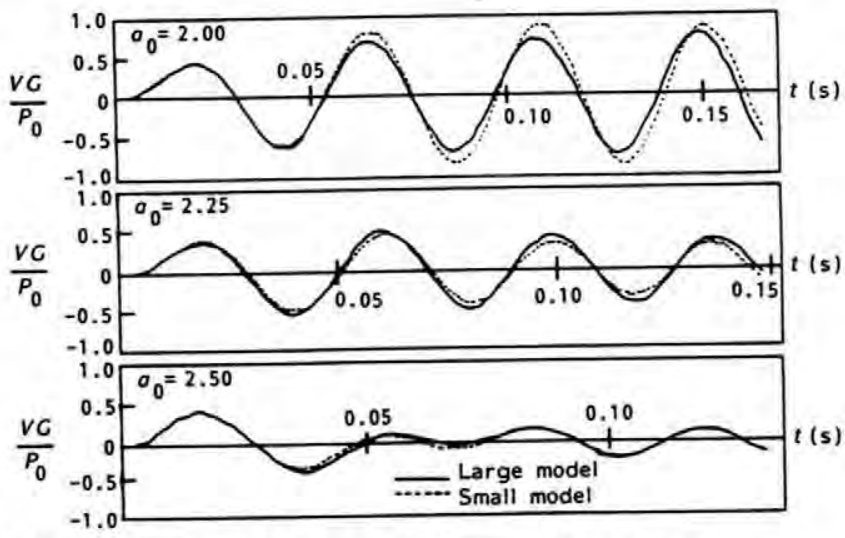

Figure 3 Normalized vertical displacement versus time of the results obtained with both models can be observed. This illustrates the validity of the proposed transmitting boundary for the analysis of transient problems with non-planar waves.

\section{Rayleigh waves excitation}

In the finite element mesh of the structure shown in Figure 4 , the transmitting boundaries valid for time domain analysis are placed at both sides of the model. A travelling harmonic wave of Rayleigh type will produce a system of forces acting along the boundaries. These forces represent the effects from the soil outside the boundaries on the model. As has been noted before, these forces can be computed from the free-field motions as follows:

$$
f(\omega)=\left[K_{\mathbf{R}}(\omega)+K_{\mathrm{L}}(\omega)\right] u(\omega)
$$

where $f(\omega)$ is the vector of forces applied to the boundary (nodes along line $\mathrm{A}-\mathrm{A}$, as shown in Figure 5) that produce
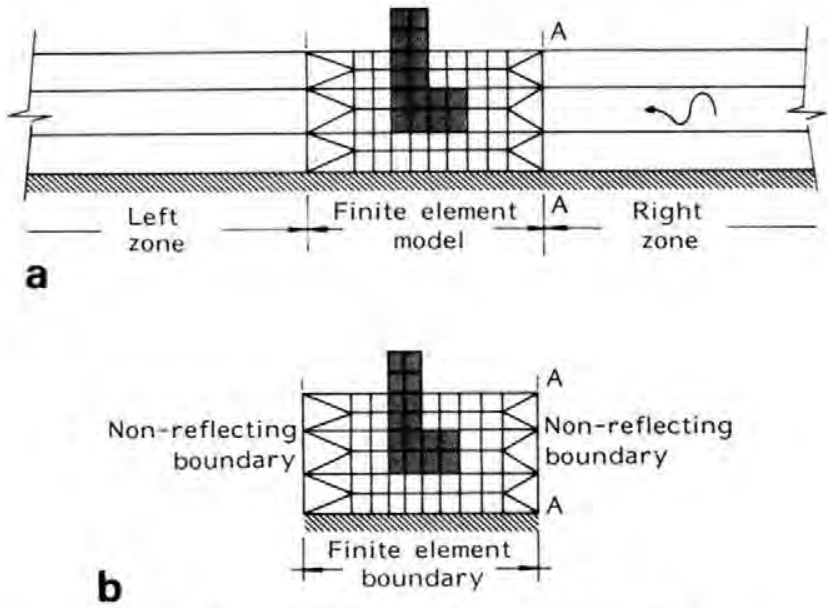

Figure 4 Soil-structure interaction finite element model
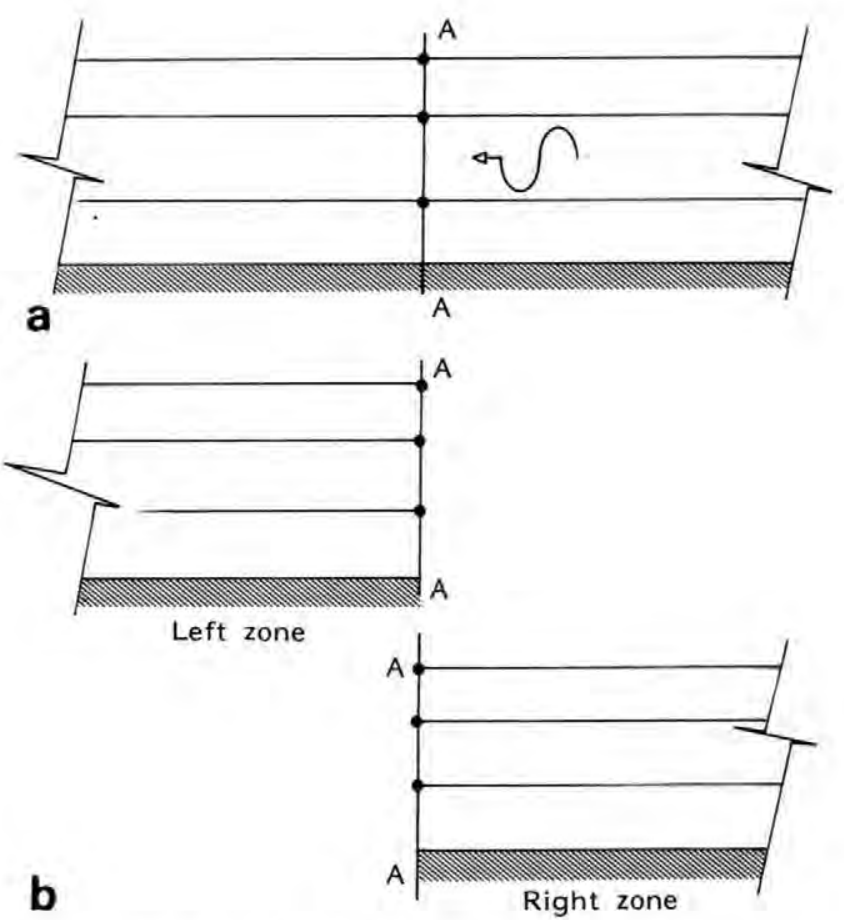

Figure 5 Boundaries showing nodes along line A-A 
free-field displacements; $u(\omega)$ are the free-field displacements at the boundaries (nodes along line $\mathbf{A}-\mathbf{A}$ ) when a harmonic wave of frequency $\omega$ is travelling through the soil; $K_{\mathrm{R}}(\omega)$ and $K_{\mathrm{L}}(\omega)$ are the consistent transmitting boundary matrices developed by Waas ${ }^{2}$ for the right and left parts of the soil, respectively, as divided in the model.

Once the forces $f(\omega)$ have been obtained, the corresponding values in the time domain can be computed by the expression

$$
f(t)=\operatorname{Re}\left[f(\omega) \mathrm{e}^{\mathrm{i} \omega t}\right]
$$

This technique could be extended to handle nonharmonic excitation, using the results of the Fourier transform for both steady state and transient situations.

\section{Dynamic earth pressures developed by a soil against a retaining wall}

In order to show the applicability of the ideas presented above, the dynamic earth pressures against a retaining wall problem, taking into account the action of Rayleigh waves and surcharge effects, are analysed. The finite element model used is shown in Figure 6. It should be pointed out that a non-reflecting boundary exists on the right side of the model. The Rayleigh waves travel from the right to the left. The wall was considered as rigid, and a viscoelastic soil behaviour with an internal soil damping ratio $\delta=0.05$, shear modulus $G=18000 \mathrm{t} \mathrm{m}^{-2}$ and Poisson ratio $v=0.4$ was supposed.

The dynamic equilibrium equations for all the analyses were solved in the time domain by direct integration procedures. In this paper the Wilson- $\theta$ method is used. This technique makes it possible to obtain the dynamic earth pressure distribution during the transient regime.

\section{Rayleigh wave excitation}

First, harmonic Rayleigh waves, with angular frequency $\omega$ and period $T$, propagating in a horizontal direction, are considered. These waves induce a horizontal peak acceleration of about $0.1 g$, where $g$ represents the acceleration of gravity.

The dimensionless frequency $a_{0}$, in this case defined as $\omega h / \beta, h$ being the wall height and $\beta$ the shear wave velocity, is varied between 1.57 and 5.0 , with the first value corresponding to the fundamental frequency of the soil layer.

In all of the cases analysed, the stationary regime of the vibratory process was reached after a few cycles. It is interesting to note that this number of cycles increases with increased dimensionless frequency $a_{0}$.

The horizontal and vertical thrust components and the overturning moment, obtained during the transient and

Non-reflecting

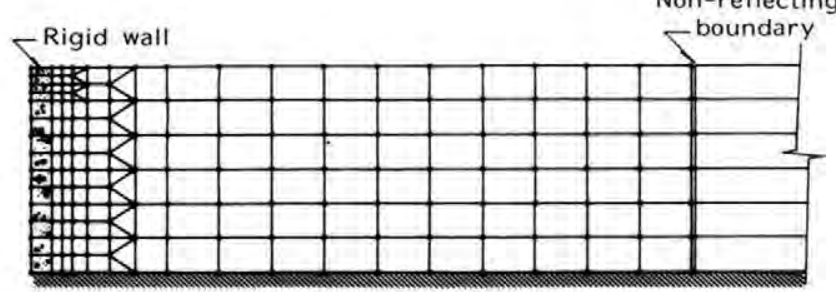

Figure 6 Dynamic finite element model
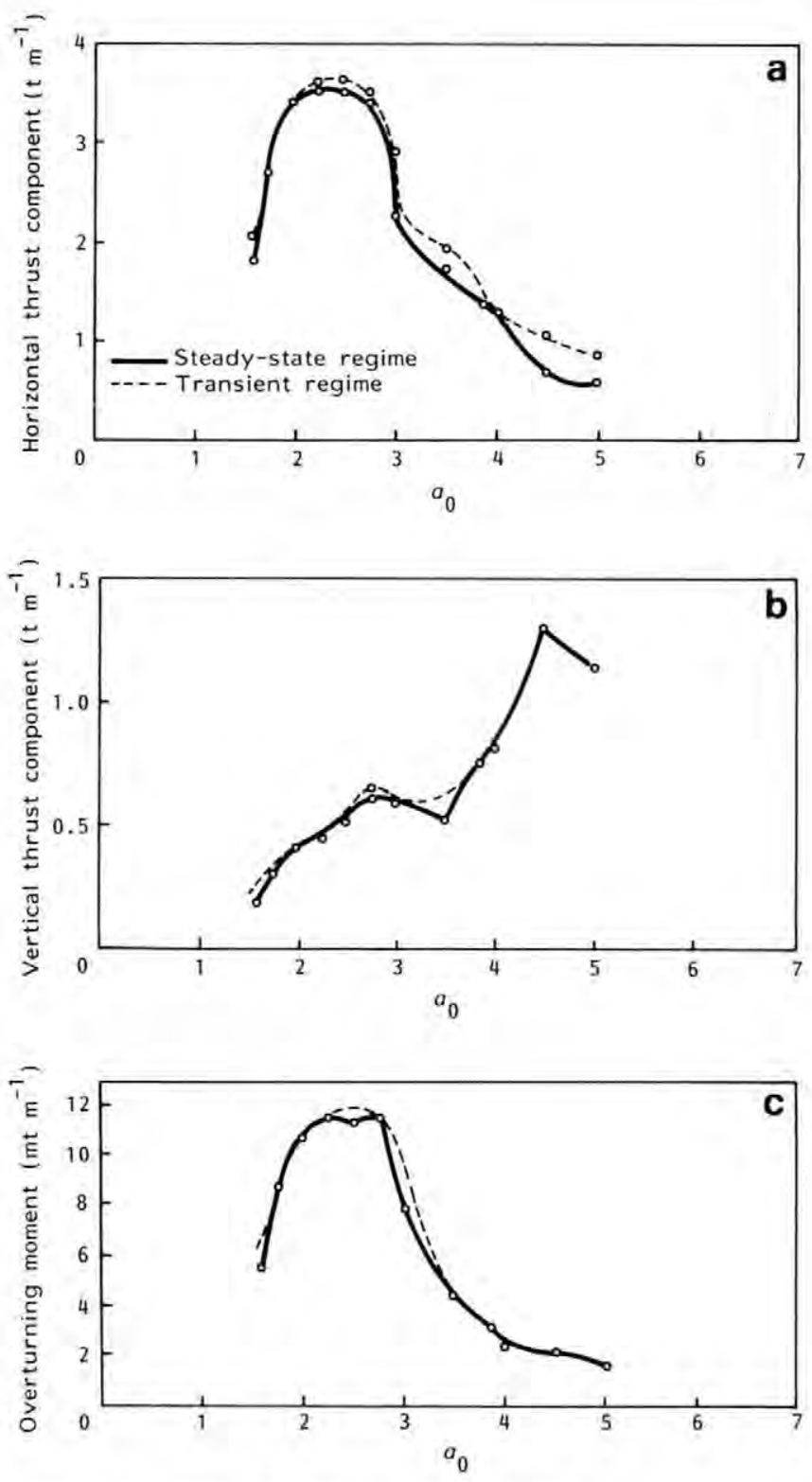

Figure 7 Results of Rayleigh wave hypothesis

steady state are shown in Figure 7 for several values of the dimensionless frequency $a_{0}$. Some obvious conclusions can be drawn from Figure 7, i.e. the horizontal thrust component reaches maximum value when $2<a_{0}<$ 2.75 , and decreases with $a_{0}>2.75$. This behaviour is also observed for the overturning moment. Furthermore, the vertical thrust component increases when $a_{0}<4.50$, and decreases for $a_{0}>4.50$.

Finally, it is observed that the transient response may be higher than the steady state response. The maximum value of the steady state response can be 2.7 times higher than obtained from the Mononobe-Okabe theory ${ }^{13}$, for the same hypothesis (level of excitation, characteristics of the soil, wall rigidity etc.). These facts would have to be taken into account for design purposes and it has also been observed by Gómez-Massó et al. ${ }^{14}$ for Rayleigh waves seismic excitation in a frequency-domain analysis.

In a second analysis, a static surcharge of $30 \mathrm{t} \mathrm{m}^{-2}$ was considered by adding equivalent masses at the nodes of the surface. The maximum horizontal thrust compo- 
nent, during the transient and stationary phases of the process, is shown in Figure 8.

A comparison may be made with previous results. It can be noted that the horizontal thrust component, considering static surcharge effects, may be as much as twice that produced in the first analysis, for the relative intensities of the surcharge and Rayleigh waves considered. The influence of static surcharge on pressure distribution can be observed in Figure 9. It is interesting to note that the pressure distribution with no surcharge is similar to that recommended in the Mononobe-Okabe theory ${ }^{13}$.

\section{Dynamic surcharge excitation}

In the second phase of the analysis, a dynamic surcharge of maximum value of $10 \mathrm{t} \mathrm{m}^{-2}$, varying harmonically with time, is considered by a set of time-dependent forces acting at surface nodes. The integration time steps $\Delta t$, for

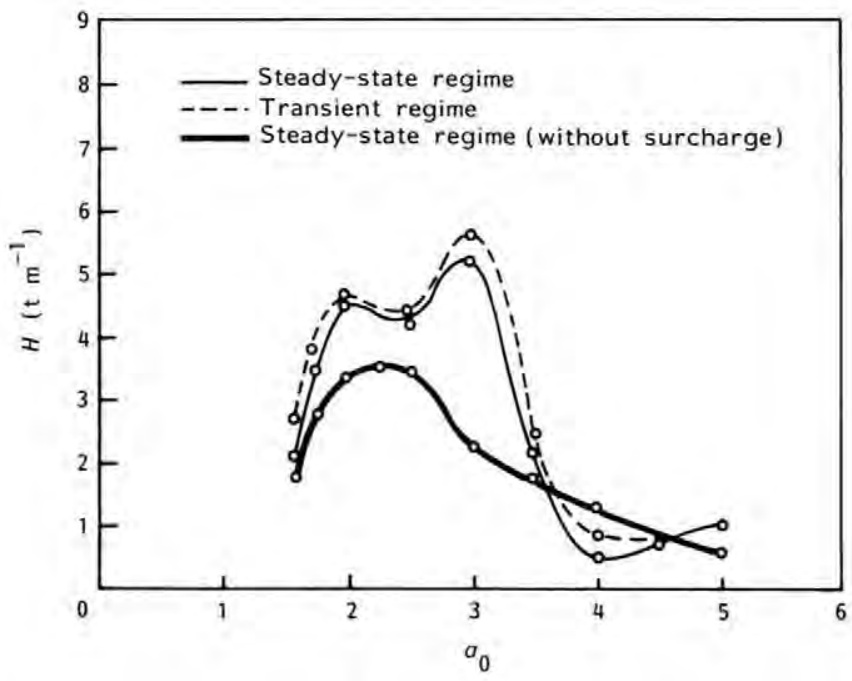

Figure 8 Influence of surcharge on horizontal thrust component

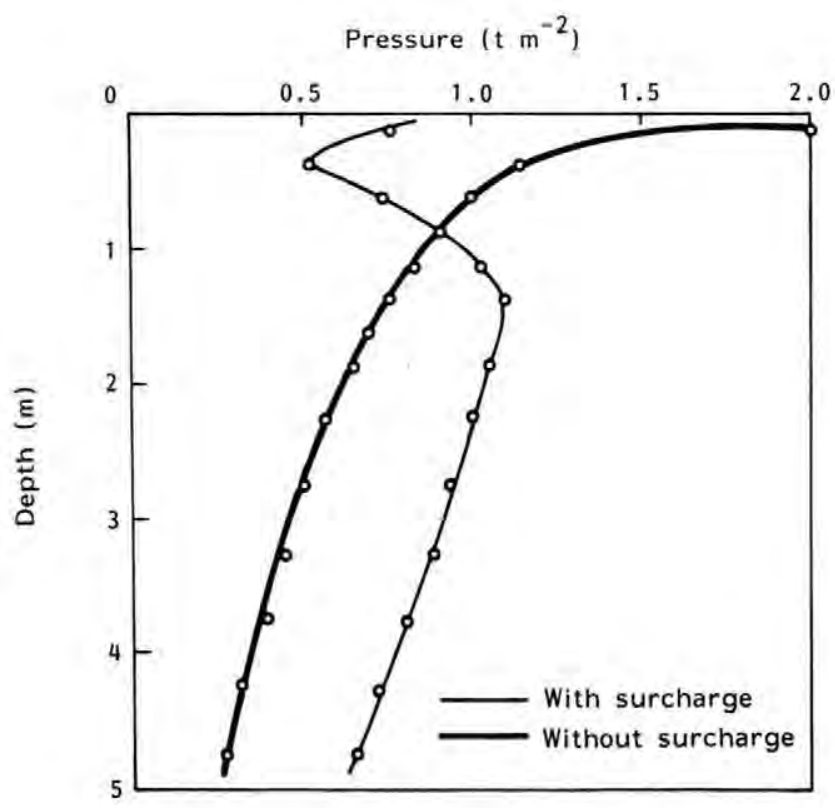

Figure 9 Pressure distributions different values of the dimensionless frequency $a_{o}$ are

$$
\begin{array}{ll}
0.25<a_{0}<1.57 & \Delta t=T / 16 \\
1.57<a_{0}<4.00 & \Delta t=T / 32 \\
4.00<a_{0}<5.00 & \Delta t=T / 64
\end{array}
$$

where $T$ is the surcharge period.

As in the previous analyses, the system reaches the stationary regime after only a few cycles. In Figure 10
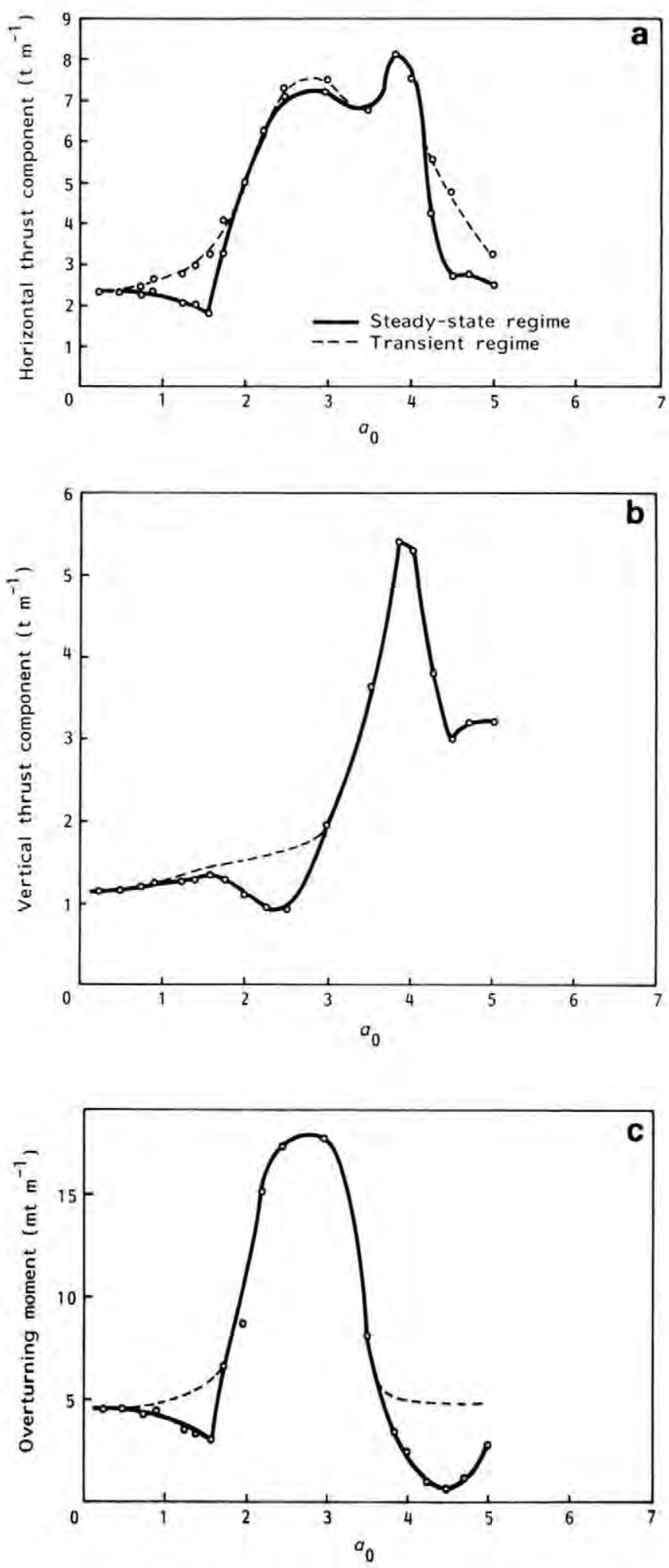

Figure 10 Results of dynamic sucharge action 
the computed results are shown. The horizontal thrust component in the transient regime decreases with increased $a_{\mathrm{o}}$. A minimum value of this component is obtained for $a_{\mathrm{o}}=1.57$ (a value that corresponds to the fundamental frequency of the soil layer system), and then it increases with increased $a_{\mathrm{o}}$. The maximum value of this component is reached when $a_{\mathrm{o}}=3.85$ (fundamental dimensionless vertical frequency of the system).

Finally, it is shown that the transient horizontal thrust component could be twice that produced during the stationary regime. This occurs when $1<a_{0}<2$ and $4<a_{0}<5$.

\section{Conclusions}

A method for the treatment of harmonic soil-structure interaction problems has been presented. Two main points have been considered; first, the formulation of non-reflecting boundaries placed at the two sides of the model; second, the technique of introducing excitation in the finite element model.

The method can be applied to different harmonic problems of interaction between soil and structure, such as the vibration effects of rotating machines. An example has been presented, namely, the study of the dynamic earth pressure developed by a soil against a retaining wall, when Rayleigh waves and static and dynamic surcharges are acting. In this example only viscoelastic materials for the soil are considered, so the results should be interpreted in this framework. However, the method can also be applied to nonlinear problems since the equations of the motion are solved by a step by step procedure in the time domain.

Finally, it is also possible to solve transient problems with this methodology. In the examples studied, the importance of these transient regimes is observed. In some cases the values reached during the transient phase are much greater than those produced during the steady state regime. This fact can be very important from the structural design point of view.

\section{References}

1 Bayo, E. and Wilson, E. L. 'Numerical techniques for the evaluation of soil-structure interaction effects in the time domain', Earthquake Eng. Res. Center Rep. No. UCB/EERC-83/04, 1983

2 Waas, G. 'Linear two-dimensional analysis of soil dynamic problems in semi-infinite layer media', Ph.D. Thesis, University of California, Berkeley, 1972

3 Navarro, C. and Samartin, A. 'A transmitting boundary in time domain for soil-structure interaction problems', Transient/dynamic analysis and constitutive laws for engineering materials. Vol. 2, Martinus Nijhoff Publishers, 1987

4 Navarro, C. and Samartin, A. 'The use of non-reflecting conditions of soil dynamics', 10th Int. Conf. Boundary Element Method. Southampton, 1988

5 Lysmer, J., Udaka, T., Tsia, C. F. and Seed, H. B. 'FLUSH: A computer program for approximate 3-D analysis of soil-structure interaction problems', Earthquake Eng. Res, Center Rep. No. EERC $75-30,1975$

6 Gómez-Massó, A. 'CREAM, A computer program for generalized soil-structure interaction dynamic analysis', 1979, unpublished

7 Zienkiewicz O.C., Bicanic, N. and Shen, F.Q. 'Single step averaging generalised Smith transmitting boundary in computational dynamics'. Transient/dynamic analysis and constitutive laws for engineering materials, Vol. 2, Martinus Nijhoff Publishers, 1987

8 Shen, F. Q. 'Transmitting boundary in seismic soil-structure interaction', Ph.D. Thesis, Swansea University, 1988

9 Smith, W. 'A non-reflecting boundary for wave propagation problems', J. Comp. Phys. 1973, 15

10 Wolf, J. P. 'A comparison of time-domain transmitting boundaries', Earth. Eng. Struct. Dyn. 1986, 14

11 Tassoulas, J. L. and Kausel, E. 'Elements for the numerical analysis of wave motion in layered strata', Int. J. Num. Meth. Eng. 1983, 19

12 Alheid, H. J., Sarfeld, W., Honeker, A. and Zimmer, H. 'Transmitting boundaries in time domain for 2-D nonlinear analyses of deep underground structures', Proc. NUMETA 85, 1985

13 A.S.C.E. 'Structural analysis and design of nuclear power plant facilities', Ch. 7, 1980

14 Gomez-Masso, A., Chen, J. C., Pecker, A. and Lysmer, J. 'Seismic pressure on embedded structures in different seismic environments', Soil dynamics and earthquake engineering conferences, Southampton, 1982 